\title{
Anti-PD-L1 Monoclonal Antibody LY3300054
}

National Cancer Institute

\section{Source}

National Cancer Institute. Anti-PD-L1 Monoclonal Antibody LY3300054. NCI Thesaurus.

Code $C 128138$.

A monoclonal antibody directed against programmed cell death-1 ligand 1 (PD-L1) with immune checkpoint inhibitory and potential antineoplastic activities. Upon administration, anti-PD-L1 monoclonal antibody LY3300054 binds to PD-L1 and prevents the interaction of PD-L1 with its receptor programmed cell death protein 1 (PD-1). This inhibits the activation of PD-1 and its downstream signaling pathways, which may enhance the Tcell-mediated immune response to neoplasms and reverse T-cell inactivation. PD-L1 is overexpressed by many human cancer cell types. PD-L1 binding to PD-1 on T-cells suppresses the immune system and results in immune evasion. PD-1, a transmembrane protein expressed on activated T-cells, is a negative regulator of the immune system that limits the expansion and survival of CD8+ T-cells. 